\title{
Can we improve on Wilson and Jungner's principles of screening for disease?
}

\author{
Robert A. Smith PhD
}

- Cite as: CMAJ 2018 April 9;190:E414-5. doi: 10.1503/cmaj.180330

See related article at www.cmaj.ca/lookup/doi/10.1503/cmaj.171154

W ilson and Jungner's Principles and Practice of Screening for Disease - which was published 50 years ago and is number 34 in the World Health Organization's Public Health Papers series - may be the most widely referenced work in the screening literature. ${ }^{1}$ Citing Wilson and Jungner's principles in any article or book chapter that discusses the knowledge and conditions that must be in place to consider mass screening is de rigueur, although citations commonly derive from secondary sources and are usually ceremonial in nature.

In linked research, Dobrow and colleagues set out to review published work since 1968 that addressed principles and prerequisites for population-based screening to determine if and how Wilson and Jungner's principles have evolved. ${ }^{2}$ The authors conducted a systematic review, identifying 41 publications and, from them, 367 unique principles related to screening. These were grouped into 12 distinct categories of consolidated principles, which were then grouped into three domains: disease/ condition, test/intervention and program/system principles. They then engaged screening experts in a two-round Delphi process to assess the level of agreement with these principles and to further refine them. Wilson and Jungner's original 10 principles, which the pair described as "a number of points that might be regarded as guides to planning case-finding," ${ }^{1}$ are reordered so as to cluster them logically, and the 12 refined principles are presented alongside them. The refined principles show an evolution in thinking about principles and practice of screening for disease that reflects mainly a shift from greater emphasis on the disease and its detection, diagnosis and treatment, to program and system principles. This shift in emphasis is very likely due to the accumulation of experience with opportunistic and programmatic screening over the past five decades.

Remarkably, the findings of the linked study, which offer the most comprehensive list of screening principles to date, underscore how little coordinated international leadership there has been to evolve and refine Wilson and Jungner's principles since they were published. Thus, it is not surprising that individuals and some institutions, left to their own devices, would seek to supplement a principle with greater specificity, or add one that they believe is important. Indeed, the original 10 principles invite

\section{KEY POINTS}

- Wilson and Jungner's small book Principles and Practice of Screening for Disease was published 50 years ago.

- New research refines the original 10 principles, adding new principles that amount to a postimplementation guide outlining the conditions that must be in place for a screening program to be successful and to evolve successfully.

- Evaluations of established screening programs show numerous, enduring shortcomings related to screening adherence rates, quality assurance, communication and equality of access.

- The refined principles may be considered both as a planning guide and a checklist to measure the degree to which a new screening program is making reasonable progress to fulfilling these objectives.

this greater specificity. Yet lack of specificity also allows for variation in what is minimally acceptable across diseases and screening program parameters.

A basic lecture on screening will note that it is difficult to see how mass screening could be justified if any one of Wilson and Jungner's 10 principles were not met, but also that the principles are vague with respect to thresholds that would allow each to be checked off, alone or in context with other principles, when considering mass screening. However, each principle is clear with respect to the need to consider and justify whether there is sufficient disease burden, opportunity for successful intervention, and an acceptable cost-effective strategy to screen a healthy population for occult disease, which is probably why these "10 commandments" have endured for so long.

The principles resulting from the Delphi process in the linked research take us further, but perhaps the evolution in thinking that the authors mined and processed with the help of screening experts is best viewed as a refinement, rather than an improvement, of Wilson and Jungner's planning guide, with the addition of a postimplementation guide to the conditions that must be in place for a screening program to be successful and to evolve successfully. In some respects, a requirement to meet all of the 12 refined screening principles before initiating a screening program would be 
unrealistic, since the infrastructure of a screening program and its integration in the broader health care system should evolve with greater experience, with infrastructure and integration adjusting to each other.

Screening can be described as a cascade of events. Years of experience with screening have shown us that the concept of a low-cost, simple, accurate test that is applied to the many without disease to discover the few with disease is an enormously complex undertaking that rarely meets these criteria. Thus, the refined principles may be considered both as a planning guide and a checklist to measure the degree to which a new screening program is making reasonable progress toward fulfilling these objectives. They could also be viewed as a benchmarking tool to evaluate mature screening programs. A review of these updated principles in the context of screening for cancer is a reminder that much work remains to be done.

Wilson and Jungner's small book not only described principles of screening, but also addressed the challenges they anticipated would be faced in screening for common chronic conditions, including challenges in cervical cancer screening, which had been introduced at the time their book was written, and those that were anticipated for breast, colorectal and lung cancer screening. ${ }^{1}$ By any measure, screening for breast, cervical and colorectal cancer has been successful, and significant reductions in mortality associated with early detection are evident in settings with mature opportunistic and organized programs. ${ }^{3-5}$ Screening for lung cancer is just now being implemented in some settings and faces the same challenges that were experienced during the early phases of implementing screening for other cancers. However, evaluation of established programs also shows numerous, enduring shortcomings related to screening adherence rates, quality assurance, communication and equality of access.

The 12 refined screening principles reported in the linked article are a categorical summary for measuring how well we're applying existing knowledge and the challenges that remain when knowledge is lacking. The study's authors call for ongoing attention to the principles and practice of screening, and their refined principles represent a foundation for new, and hopefully regular, consideration of how we should view the implementation, management and evaluation of programs for cancer screening. It is likely that the agreement reached during the study's Delphi process will not be entirely shared by everyone involved with screening, and that national systems will put greater or lesser emphasis on some of these principles. But the study authors call our attention to the fact that, after 50 years of experience, Wilson and Jungner's principles needed a fresh look. Dobrow and colleagues have made an important contribution to the screening literature, and it is very likely that we'll begin to see two citations, 50 years apart, when describing the principles and practice of screening for disease.

\section{References}

1. Wilson JMG, Jungner G. Principles and practice of screening for disease. Geneva: World Health Organization; 1968.

2. Dobrow MJ, Hagens V, Chafe R, et al. Consolidated principles for screening based on a systematic review and consensus process. CMAJ 2018;190:E422-9.

3. Lauby-Secretan B, Scoccianti C, Loomis D, et al.; International Agency for Research on Cancer Handbook Working Group. Breast-cancer screening viewpoint of the IARC Working Group. N Engl J Med 2015;372:2353-8.

4. Peirson L, Fitzpatrick-Lewis D, Ciliska D, et al. Screening for cervical cancer: a systematic review and meta-analysis. Syst Rev 2013;2:35.

5. Lauby-Secretan B, Vilahur N, Bianchini F, et al.; International Agency for Research on Cancer Handbook Working Group. The IARC perspective on colorectal cancer screening. N Engl J Med. In press.

\section{Competing interests: None declared.}

This article was solicited has not been peer reviewed.

Affiliation: Cancer Control Department, American Cancer Society, Atlanta, Ga.

Correspondence to: Robert A. Smith, rsmith@cancer.org 